\title{
AN ANALYTICAL COST MODEL FOR INVESTMENT CASTING
}

\author{
M. Mandolini ${ }^{1, \bigotimes}$, F. Campi ${ }^{1}$, C. Favi ${ }^{2}$, P. Cicconi ${ }^{1}$ and M. Germani ${ }^{1}$ \\ ${ }^{1}$ Università Politecnica delle Marche, Italy, ${ }^{2}$ University of Parma, Italy \\ $\triangle$ m.mandolini@univpm.it
}

\begin{abstract}
Analytical cost estimation of investment casted products during design phase is a complex task since the quantity of parameters to be evaluated. So far, there is a short literature on such cost estimation models. This paper attempts to improve the cost model presented by Boothroyd and Dewhurst. Improvements (mainly focused on cluster assembly and investment, sintering and melting phases) were defined and verified in cooperation with two foundries. Tested on eight components, deviation between estimated and actual costs is around $14 \%$ for manual production lines and $6 \%$ for automatic ones.
\end{abstract}

Keywords: design for $x(D f X)$, design costing, process modelling, investment casting, cost estimation

\section{Introduction}

Investment casting (also known as 'lost wax casting' or 'precision casting') has been a widely used process for centuries. It is known for its ability to produce components of excellent surface finish, dimensional accuracy and complex shapes. It is especially useful for making castings of complex and near-net shape geometry, where machining may not be possible or too wasteful. The part obtained by investment casting has dimensions ranging from a few grams to $50 \mathrm{~kg}$ and from $5 \mathrm{~mm}$ to $300 \mathrm{~mm}$. The tolerance range normally achievable in investment cast casting is $\pm 1 \%$ of the nominal size, with a minimum of $\pm 0,10 \mathrm{~mm}$ for dimensions lower than $10 \mathrm{~mm}$, with a minimum roughness of $3.2 \mu \mathrm{m}$. Figure 1 shows the investment casting process phases (Pattnaik et al., 2012). Pieces manufactured by investment casting process are highly costly due to the low level of automation of the process and the quantity of phases.

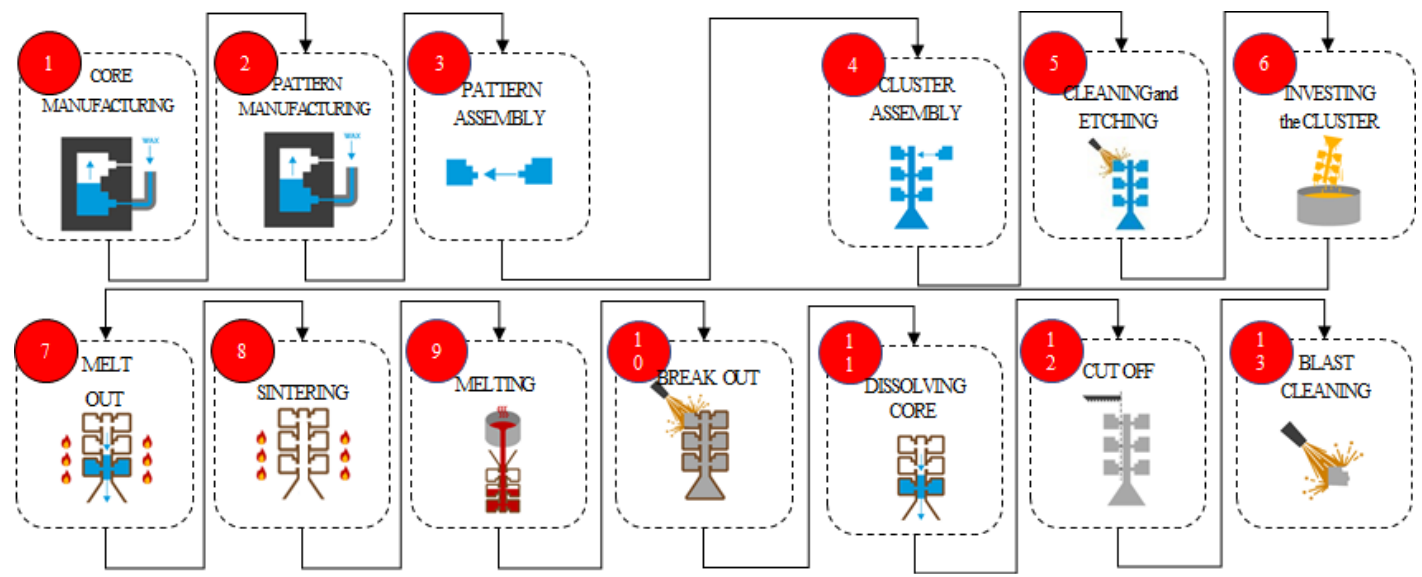

Figure 1. Investment casting process phases 
The design of investment casting products is a long and iterative process, which begins from product specification and ends with the detailed definition of technical and functional requirements for the product. It is well-known that, although product design and engineering phases consume approx. 10\% of the total budget for a new project, typically $80 \%$ of manufacturing costs are determined by such phases (Ulrich and Eppinger, 2003). Product cost estimation is commonly carried out by product and process design engineers by employing different methods. In this context, the cost model is the mathematical representation of those algorithms and data which are used for computing the product cost from its design features.

In literature, it is possible to recognize several cost models based on analogical, analytical, featurebased and parametric methods, which are used for product cost estimation. However, there are very few scientific papers on early cost estimation of wax-casted products. The work presented in this paper attempts to bridge this gap by developing an analytical cost model for investment casting cost estimation, using (Boothroyd et al., 2011) as a reference and starting point. The proposed cost model may be used by design and cost engineers for evaluating the cost for each production phase of the entire investment casting process. This paper illustrates the improvements to the reference cost model so that it can be robustly used for analytically estimating cost of lost-wax casted products, realized employing both manual and automatic production lines.

The paper is structured as follow. Section 2 presents the literature review on cost estimation methods and cost models for casting processes. Section 3 reports the main improvements established to the cost model proposed in (Boothroyd et al., 2011), thought to improve its robustness for products wax-casted using manual production lines. Section 4 presents a preliminary validation of the improved cost model, by comparing estimated values with actual ones. Lastly, section 5 summarizes the main outcomes of the proposed cost model and future developments in this field.

\section{Research background}

During the product development process (PDP), cost plays a critical role and drives most of the technical and technological solutions (Sohlenius, 1992). Cost estimation is a design task which allows a designer to evaluate the production costs of products before their manufacturing (Das et al., 2000). Cost estimation activity includes a classification of cost items both for the materials and the manufacturing processes. In addition, cost estimation requires a definition of a mathematical model (namely, cost model) which integrates the cost items (Niazi et al., 2005).

Several cost estimation approaches are available today for evaluating product cost at design stage. They can be grouped in two main families: (i) qualitative methods, which are primarily based on a comparison analysis of a new product and an existing one and, (ii) quantitative methods, which are based on a detailed analysis of a product design, including its features and corresponding manufacturing processes (Niazi et al., 2005).

Qualitative methods include intuitive (Rush and Roy, 2001), analogical (Duverline and Castelain, 1999; Wang et al., 2003; Arundacahawat et al., 2013) and knowledge based (García-Crespo et al., 2011; Shehab and Abdalla, 2002). The intuitive and knowledge-based methods are based on the experience of the estimator. The analogical method involves comparison of a new product with similar existing products. Case based reasoning has been applied to improve the results of the analogical method (Duverline and Castelain, 1999).

Quantitative methods include analytical (Favi et al., 2017; Campi et al., 2019), parametric (Farineau et al., 2001; Martinelli et al., 2019) and feature based methods (Ou-Yang and Lin, 1997; Jung, 2002). The analytical method involves the manufacturing process decomposition into elementary tasks, and empirical equations are used for estimating the cost of each one. The feature-based method uses geometric features (such as slot, hole and rib) of the product and tooling as the basis for cost estimation. The parametric cost estimation methods involve formulating relations between product characteristics and its cost using available data.

Several research works are focused on cost estimation of specific operations or domains. In relation to a technology, specific analytical cost estimation models were developed based on the analysed manufacturing process: (i) chip metal forming (Boothroyd and Radovanovic, 1989), (ii) hole making 
(Luong and Spedding, 1995), (iii) sheet metal (Verlinden et al., 2008), (iv) injection moulding (Fagade and Kazmer, 2000) and (v) forging (Martinelli et al., 2019).

For casting processes, different models have been developed based on the process's peculiarities. Several researchers (Chronister, 1975; Kulkarni, 1988) have identified the major cost elements of a casting process, such as material, tooling, labour, energy and overheads. In practice, many foundries and their customers still estimate cost based on component weight, corrected for the expected level of production difficulties, scrap and yield. The method works well in mass production foundries making castings of similar characteristics, but it is not suitable for job shop or batch type foundries that have a wide range of products. To alleviate these problems, more elaborate cost models have been proposed by identifying and calculating the detailed cost elements (Sajid et al., 2018; Maciol, 2017).

However, very few works have been reported on cost estimation of investment casting. Creese (Creese and Adithan, 1992) developed a simplified parametric model for calculating the cost of investment casting products during initial design stage.

The DFM Concurrent Costing ${ }^{\circledR}$ software developed by Boothroyd Dewhurst Inc. (Boothroyd et al., 2011) has an analytic and detailed investment casting cost estimation module aimed at product designers. It considers most phases of the process (pattern and core manufacturing, pattern and cluster assembly, cleaning and etching, investment operation, melting, sintering, break out, blast cleaning and cut off), but the default process parameters seem to be more focused on automatized plants and, for example, it does not consider the adaptation of the process times when changing type of production line (i.e.: from automatic to manual and vice-versa).

\section{Improvement of reference model}

The analytical cost model for investment casting, developed in this work, is an improvement of that one proposed by Boothroyd and Dewhurst (Boothroyd et al., 2011) and taken as reference. The approach for this improvement consists of four steps, hereafter summarized:

1. Formalization of the reference cost model into an electronic spreadsheet;

2. Workshops with foundries for analysing the manufacturing process and gathering useful data;

3. Improvement of the reference cost model;

4. Validation of the improved cost model.

The first step consisted in collecting equations and data available in (Boothroyd et al., 2011). This set of information was integrated with other data (i.e.: pattern materials, cast materials, wax injection presses and furnaces) retrieved from "DFM Concurrent Costing" (By Boothroyd Dewhurst, Inc.). The result consisted in an electronic spreadsheet to be used for preliminary estimating the manufacturing cost for investment casting. Hereafter, this result will be called DFMA.

After that, twenty components (different for material, weight and realized with single patterns) were selected in cooperation with two foundries, with the aim to evaluate the most cost expensive investment casting phases. The result, which is shown in Figure 2, allowed authors to focus the analysis on core manufacturing (9-10\% of the total manufacturing cost), pattern manufacturing (9$29 \%$ ), investing the pattern cluster (11-15\%) and melting phases (37-54\%), which globally represent the $87-90 \%$ of the product cost. To be noted that the melting phase accounts material cost, while tooling cost is excluded from this analysis.

The second step consisted in organizing two workshops with the selected foundries, equipped with manual (the first foundry) and automatic (the second foundry) production lines. The aims of these workshops were: (i) present data elaborated in the previous step, (ii) discuss with expert production engineers about the most important product and process cost drivers and (iii) gather the most relevant process data (e.g.: standard times, cost rates, equipment, materials). For each of the previous identified process phases, the working group (consisting of authors and process experts of the involved foundries) have compared the process cost drivers assumed by DFMA with the actual ones, with the aim to establish, if required, improvements to algorithms and data. Hence, hereafter, authors present only equations and data that were revised respect the original DFMA cost model. These ones are the result of interviews and analyses of historical data of the involved foundries. 


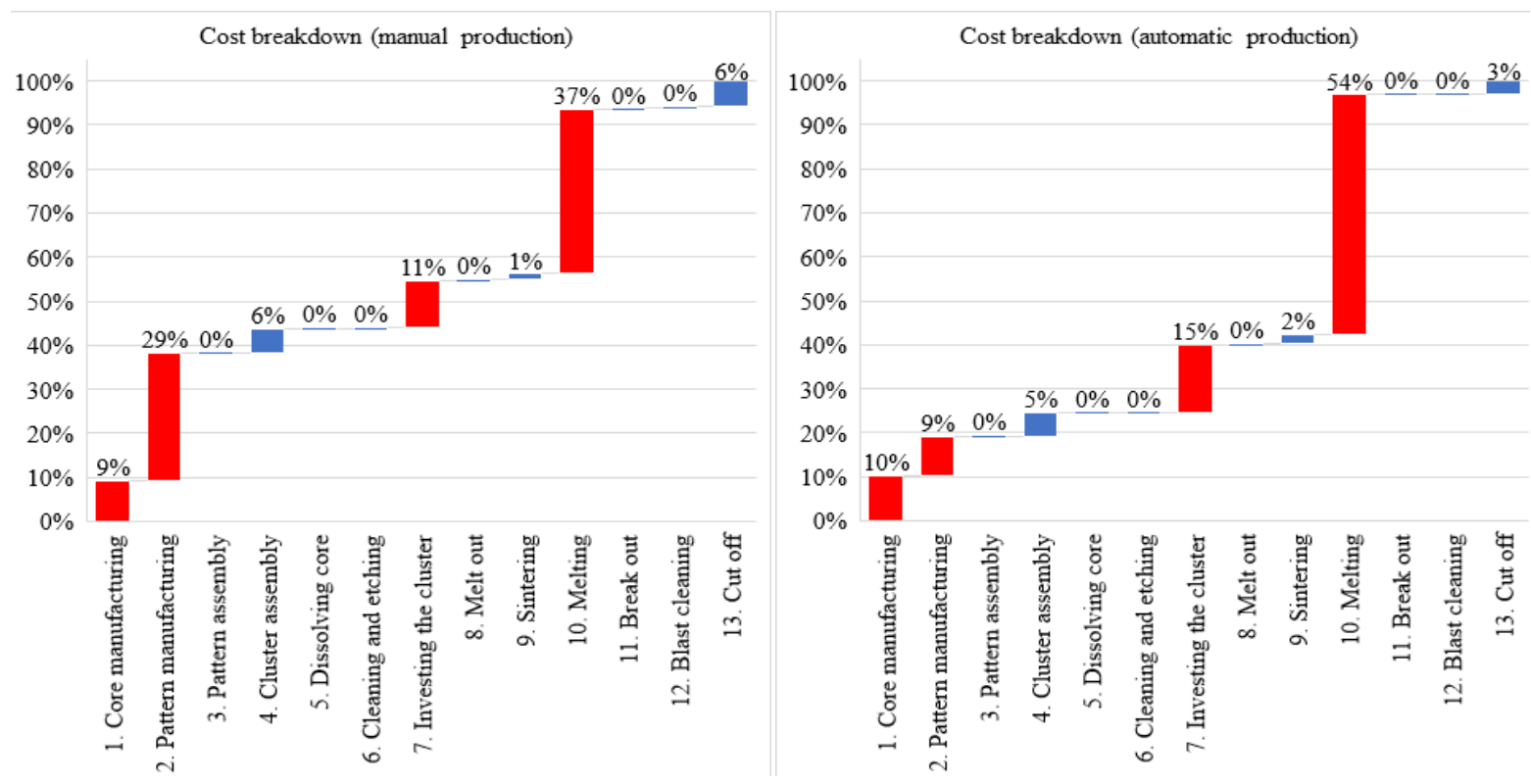

Figure 2. Investment casting cost breakdown (elaborated from Boothroyd et al., 2011)

The core and pattern manufacturing phases were jointly analysed because they were realized with the same machines (they differ only for the kind of wax used). For this process, the improvements to DFMA approach were about the wax cooling time (Equations 1 and 2) and wax injection machine setup time (this time was missing in DFMA).

CoolingTime $=$ CoolingFactor $\cdot$ SurfaceFactor $\cdot$ ToleranceFactor $\cdot$ BaseCoolingTime

$$
\text { BaseCoolingTime }=\left(3+\frac{\text { Thickness }^{2}}{\pi^{2} \cdot \text { ThermalDiffusivity }^{\prime}} \cdot\left|\ln \frac{\pi \cdot(\text { ExtractionTemperature }- \text { MoldTemperature })}{4 \cdot(\text { InjectionTemperatura }- \text { MoldTemperature })}\right|\right)^{\text {ThicknessFactor }}
$$

Cooling time depends on pattern surface quality, tolerance and thickness, on pattern material thermal diffusivity, extraction temperature, injection temperature and mould temperature. Surface factor, used for accounting the kind of mould used, is 1.4 in case of external sliders in the mould and 0.0 otherwise. Tolerance factor, used for accounting the quality of the pattern to be realised, is 1.3 in case of a tolerance less than $0.005 \mathrm{~mm}, 1.25$ when tolerance is less than $0.05 \mathrm{~mm}, 1.1$ when tolerance is less than $0.1 \mathrm{~mm}$ and 1.0 otherwise. Thickness factor, used for adjusting Equation (2) according to the pattern thickness, ranges between 1.0 for thin-walled patterns (thickness less than $1.0 \mathrm{~mm}$ ) to 0.5 for thick-walled patterns (it is 0.80 for a $20 \mathrm{~mm}$ thick pattern, 0.75 for $40 \mathrm{~mm}, 0.71$ for $80 \mathrm{~mm}$ and 0.5 otherwise).

The other manufacturing phase analysed was the pattern investment. The list of improvements to DFMA consists of many aspects, with the aim to correlate process cost drivers to product ones. The quantity of primer coats (it is fixed to 3 in DFMA) depends on the component surface roughness (the higher the quality, the higher the quantity of primer coats). It is 2 for a roughness greater or equal to $6.3 \mu \mathrm{m}, 3$ otherwise. The quantity of backup coats (it is fixed to 5 in DFMA) depends on the component weight (the higher the weight, the higher the quantity of backup coats). It is 4 for components lighter than $4 \mathrm{~kg}, 6$ for a weight greater than $10 \mathrm{~kg}$ and 5 otherwise. The investment time was deeply revised (in DFMA it is fixed to 20 and 10 seconds respectively for primer and backup coats). In the proposed cost model, this time was set to 1.2 minutes for a robotic handling and 2.4 minutes for a manual handling of the cluster (it is independent on the kind of coat). The shell mould material cost (in DFMA it is $1.00 \$$ for one kilogram of shell mould) depends on the component weight, cluster dimension and mould yield. In the proposed approach, the cost was simplified considering $2.00 €$ each coat and each cluster. This is the result of a regression analysis carried out on multiple clusters with different dimensions.

The cost model for melting phase, proposed in DFMA, was not subject to significant modifications. However, it allowed authors to deep dive the algorithms and data used for estimating the cluster 
assembling, that phase when patterns are assembled in a cluster. According to DFMA, the latter is created by assembling patterns starting from a semi-finished element, a conical pouring cup with a single stem (Figure 3.a). During the workshops with foundries, it was possible to notice that also another kind of pouring system may be used (rectangular pouring cup with multiple stems, Figure 3.b).

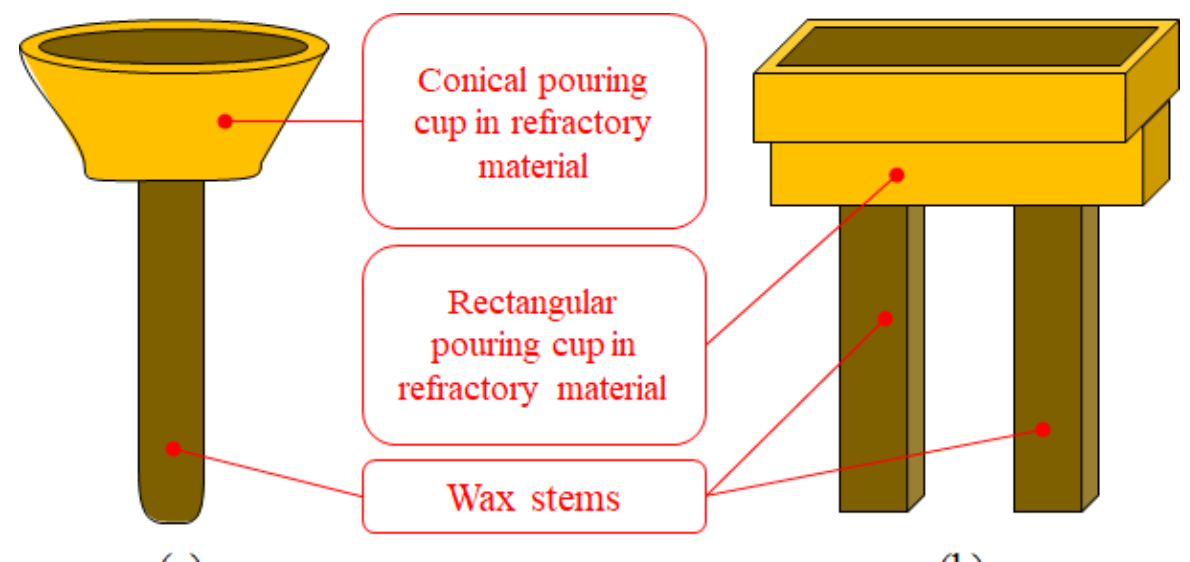

(a)

(b)

Figure 3. Two pouring systems used: (a) conical pouring cup, (b) rectangular pouring cup

For estimating the dimensions and cost of a pouring system, a database was firstly established. Each system is characterized by its dimensions (i.e. small, medium and big for conical pouring cups and 2, 3 and 4 stems for rectangular ones). The wax volume ranges between 2 and $4 \mathrm{dm}^{3}$, respectively for the small and big pouring systems. The cost of each system is estimated considering materials usage (i.e.: refractory cup and wax) and the manufacturing process (i.e.: injection of the wax within the pouring cup). The overall cost is estimated between 18.00 and $26.00 €$. Once defined the database, the pouring system is selected according to the pattern volume (the conical pouring cup is used for big components, generally greater than $1 \mathrm{dm}^{3}$ ) and quantity of pattern each cluster (i.e.: the higher the pattern quantity for each cluster, the smaller the components, the higher the quantity of wax stems). For rectangular pouring system, it is assumed that a two stems system is used for clusters with equal or less than 4 patterns, four stems system is used with clusters with more than 10 patterns. The quantity of patterns in a cluster mainly depends by its maximum weight (pouring cup and shell mould weight), which depends on the type of production lines. This is $15 \mathrm{~kg}$ for manual lines (clusters are manually handled by operators until melting) and $40 \mathrm{~kg}$ of automatic ones. The equation used for computing the cluster dimension is the same one proposed in DFMA.

The cluster assembly phase, even if it is outside the Pareto analysis presented in Figure 2, was improved since a new pouring system was introduced. Furthermore, DFMA estimates the cluster assembly time considering 20 seconds each pattern, which is a constant value that does not depend on pattern weight and quantity of gates per pattern. In the proposed cost model, the assembly time for each gate is 0.4 minutes each kilogram of casted component. Indeed, the bigger the pattern, the higher the assembly time, because the wider is the pattern surface to be heated and glued to the pouring system. The quantity of gates each pattern is evaluated through a thickness analysis of the 3D model of the pattern. Generally, a gate is located close to isolated volumes with the aim of feeding this one until the complete solidification of the component.

During the workshops with foundries, it was investigated also the cluster sintering phase, even if it was not included in the Pareto analysis. Indeed, the sintering phase may take up to three hours at $1100^{\circ} \mathrm{C}$, in electric or gas furnaces able to contains multiple clusters. DFMA assumes a sintering cost that is around $0.61 \$$ /cluster. However, the high energy consumption of such furnaces and the industrial practice suggests a greater cost. Assuming two hours for sintering a cluster, a furnace able to contains, at the same time, 5 clusters, with a rate of $30.00 € /$ hour (Italian average labour rate) and $75 \%$ of efficiency, and the involvement of one operator with an engagement of 50\% (the other $50 \%$ of time is spent for loading and unloading clusters from autoclave and puttying clusters with stucco), for a manual production line, the sintering cost is around $20.00 € /$ cluster. 
During the third step of the previously presented approach, all the improvements to the investment casting cost model proposed in DFMA have been integrated in the electronic spreadsheet which was developed at phase 1. In this manner, it was possible to draft an update the cost breakdown for investment casting (Figure 4). It is possible to observe that, according to this cost model, the most cost expensive phases are pattern manufacturing (12-20\%), cluster assembly (11-12\%), investing pattern cluster (14-15\%), sintering $(\sim 11 \%)$ and melting (32-39\%), which globally represents the $87-90 \%$ of the product cost.

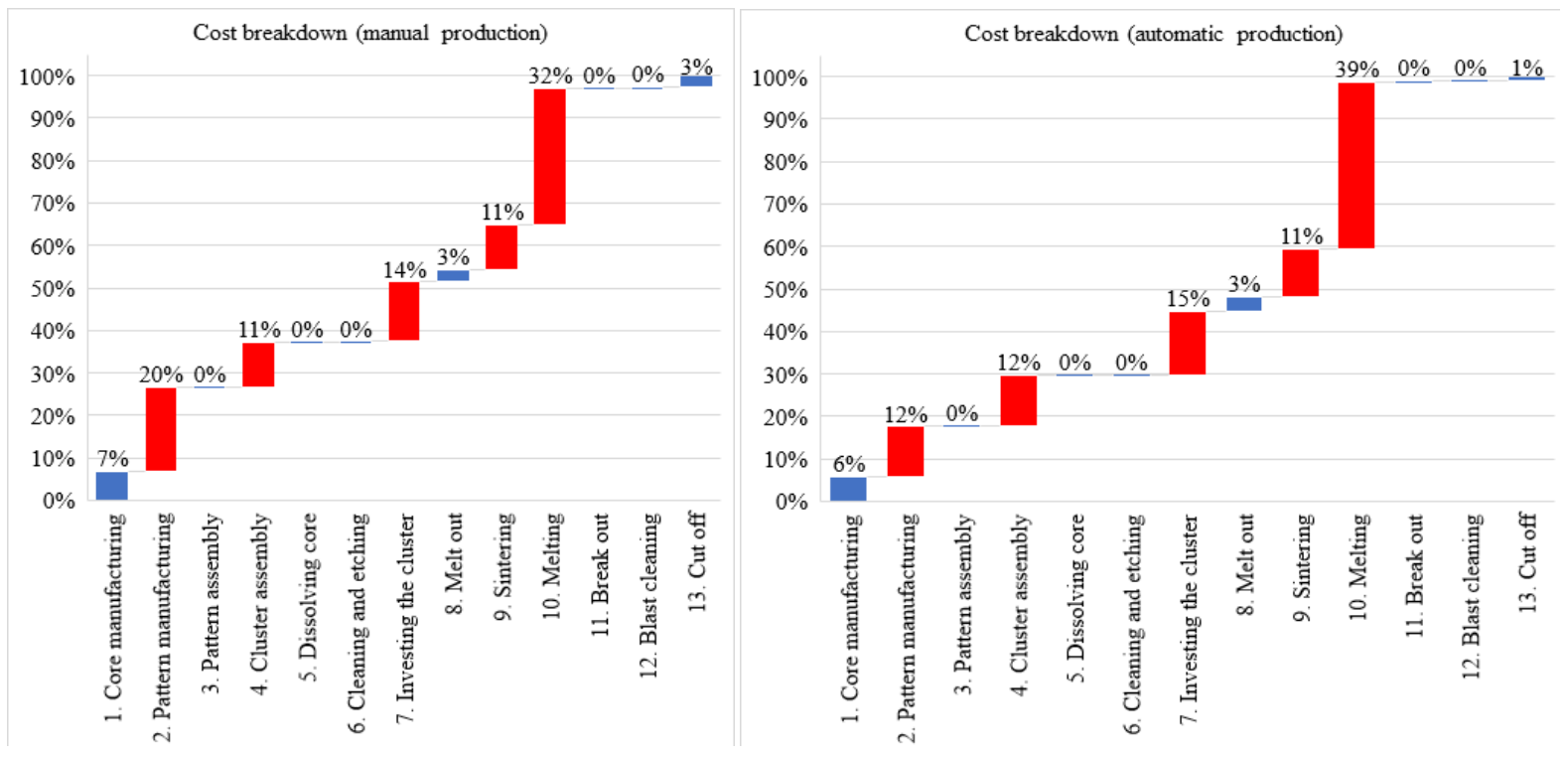

Figure 4. Cost breakdown for investment casting proposed in this work

Based the proposed cost model, two sensitivity analyses (Figure 5) were performed for evaluating the relationships between the product and process cost drivers (e.g.: part volume, thickness, area, raw material price, labour prices) with the process and raw material costs. Such analyses highlight the most important cost drivers for raw material cost (part volume, raw material price and material density) and process cost (part volume, labour prices, part thickness, part area and production batch). This result will be used for developing (future work) a parametric cost model for investment casting.

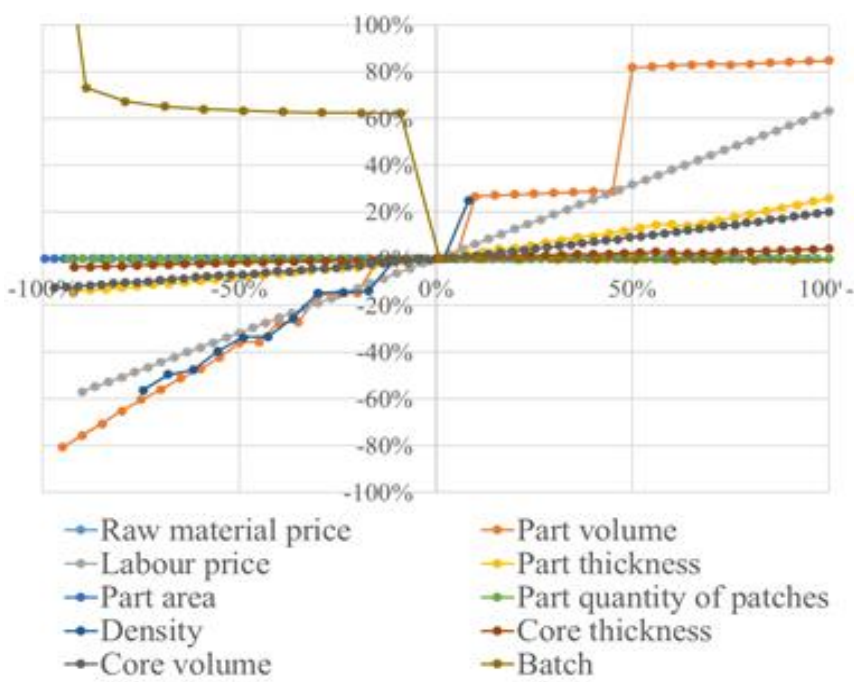

(a)

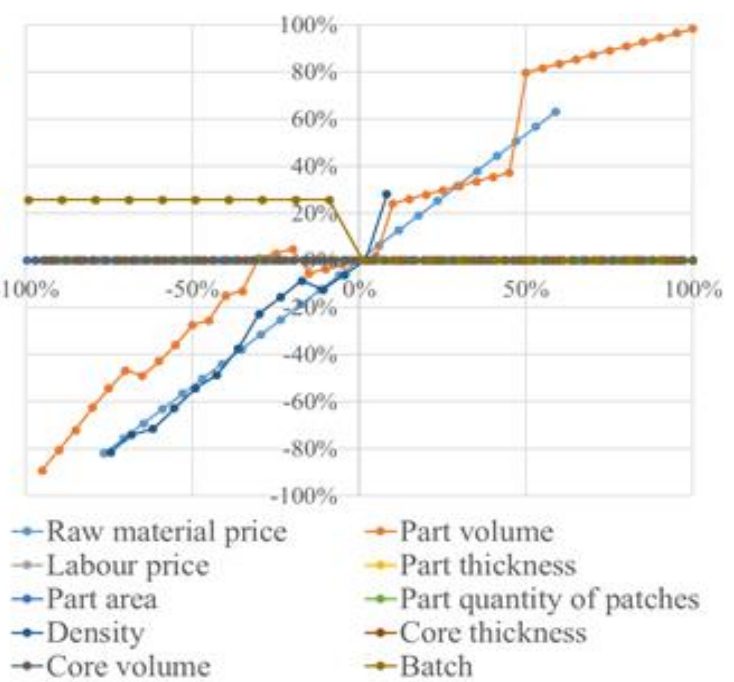

(b)

Figure 5. Sensitivity analysis for (a) process and (b) raw material cost

The cost model validation, the fourth step of the procedure, is presented in the following section. 


\section{Cost model validation}

The test aimed to evaluate the robustness of the proposed cost model, which aims to improve the DFMA reference one. The cost model presented in the previous section was used for estimating the investment casting process cost of 8 components (other than those ones used for improving the reference cost model), different in weight (from hundreds of grams to few kilograms) and materials (carbon steel and stainless steel). Results were organized in two datasets for simulating two different scenarios. In the first scenario, parts are manufactured by a foundry located in Italy and equipped with a manual line (foundry no. 1), while, in the second scenario, components are realized by a Chinese foundry employing an automatic line (foundry no. 2). Each dataset contains four groups of information, one each component. Each group consists of (i) a list of cost items estimated with the proposed model, (ii) a list of cost items estimated with the reference model and (iii) the actual total manufacturing cost provided by the involved foundries.

For each component, cost items estimated using the model here proposed were compared with those ones obtained by DFMA, while the estimated total manufacturing cost was compared with the actual one. Labour and machine rates, as well as material unitary costs, were considered as constant values (equal for each group of results) set before cost estimation.

The first dataset (manual production in Italy) consists of 4 components. For confidentiality reasons, cost values were dimensionless to the total manufacturing cost of component $1 \mathrm{~B}$, the most expensive in this scenario. From Figure 6, comparing total costs obtained with the proposed model and actual costs provided by the foundry, it is possible to observe a deviation lower than $25 \%$ for all the selected components. On the contrary, comparing actual values provided by the foundry with values obtained by DFMA, a higher deviation (> 50\%) can be noted. The average deviation from the actual cost, for the proposed cost model, is $14 \%$, better than the reference one, which is $56 \%$. Comparing the single costs items between the proposed model and DFMA, the most different phases are cluster assembly and melting. The first deviation depends by the high labour required for this phase (DFMA tends to underestimate process times and cost labour intensive phases), while the second one is influenced by the additional pouring system considered in this work.

\begin{tabular}{|c|c|c|c|c|c|c|c|c|}
\hline CO MIPO NENT NAME & \multicolumn{2}{|c|}{$1 \mathrm{~A}$} & \multicolumn{2}{|c|}{$1 \mathrm{~B}$} & \multicolumn{2}{|c|}{$1 \mathrm{C}$} & \multicolumn{2}{|c|}{ 1D } \\
\hline Material & \multicolumn{2}{|c|}{ 39NiCrMo4 } & \multicolumn{2}{|c|}{ AISI 316} & \multicolumn{2}{|c|}{ 39NiCrMo4 } & \multicolumn{2}{|c|}{ 39NiCrMo4 } \\
\hline Weight (gr) & \multicolumn{2}{|c|}{1057.00} & \multicolumn{2}{|c|}{2065.00} & \multicolumn{2}{|c|}{1408.00} & \multicolumn{2}{|c|}{1184.00} \\
\hline PRO CESS ITEM COSTS & Calc. & DFMA & Calc. & DFMA & Calc. & DFMA & Calc. & DFMA \\
\hline Core manufacturing & 1.95 & 2.00 & 2.20 & 0.48 & 1.07 & 0.47 & 2.17 & 2.68 \\
\hline Pattern manufacturing & 5.60 & 5.03 & 6.51 & 5.89 & 6.72 & 6.86 & 6.40 & 7.93 \\
\hline Pattern assembly & 0.00 & 0.00 & 0.00 & 0.00 & 0.00 & 0.00 & 0.00 & 0.00 \\
\hline Cluster assembly & 7.19 & 1.01 & 10.59 & 1.82 & 7.31 & 1.28 & 6.81 & 1.01 \\
\hline Dissolving core & 0.02 & 0.00 & 0.04 & 0.00 & 0.03 & 0.00 & 0.02 & 0.00 \\
\hline Cleaning and etching & 0.02 & 0.03 & 0.03 & 0.06 & 0.02 & 0.04 & 0.02 & 0.03 \\
\hline Investing the cluster & 8.57 & 2.55 & 15.00 & 4.66 & 10.00 & 3.28 & 8.57 & 2.76 \\
\hline Melt out & 1.87 & 0.08 & 3.27 & 0.16 & 2.18 & 0.11 & 1.87 & 0.08 \\
\hline Sintering & 6.44 & 0.29 & 11.27 & 0.59 & 7.52 & 0.39 & 6.44 & 0.29 \\
\hline Melting & 13.57 & 6.05 & 41.29 & 26.23 & 16.28 & 7.83 & 13.92 & 6.70 \\
\hline Break out & 0.12 & 0.07 & 0.21 & 0.13 & 0.14 & 0.09 & 0.12 & 0.07 \\
\hline Blast cleaning & 0.11 & 0.04 & 0.20 & 0.08 & 0.13 & 0.05 & 0.11 & 0.04 \\
\hline Cut off & 1.36 & 0.90 & 1.54 & 2.13 & 1.28 & 1.73 & 1.81 & 1.08 \\
\hline Total & 46.82 & 18.05 & 92.15 & 42.24 & 52.68 & 22.13 & 48.27 & 22.68 \\
\hline Actual value & \multicolumn{2}{|c|}{42.21} & \multicolumn{2}{|c|}{100.00} & \multicolumn{2}{|c|}{42.21} & \multicolumn{2}{|c|}{56.28} \\
\hline Dev. Total vs Actual & $11 \%$ & $57 \%$ & $8 \%$ & $58 \%$ & $25 \%$ & $48 \%$ & $14 \%$ & $60 \%$ \\
\hline
\end{tabular}

Figure 6. Foundry No. 1 components cost analysis

The second dataset (automatic production in China) contains 4 components. Even in this case, for confidentiality reasons, cost values were dimensionless to the total manufacturing cost of component 2D, the most expensive in this scenario. From Figure 7, comparing the costs obtained with the proposed 
model and the actual values provided by the foundry, for all the components it can be noted a deviation lower than $10 \%$. The same comparison gives a higher deviation for DFMA. The average deviation between the proposed cost model and actual values is $6 \%$, better than the reference one, which is $29 \%$. In this scenario, the major differences between DFMA and cost model can be found in core manufacturing and pattern assembly operations in which DFMA software considers lower values.

\begin{tabular}{|c|c|c|c|c|c|c|c|c|}
\hline COMIPO NENT NAME & \multicolumn{2}{|c|}{$2 \mathrm{~A}$} & \multicolumn{2}{|c|}{$2 \mathrm{~B}$} & \multicolumn{2}{|c|}{$2 \mathrm{C}$} & \multicolumn{2}{|c|}{ 2D } \\
\hline Material & \multicolumn{2}{|c|}{$42 \mathrm{CrMo} 4$} & \multicolumn{2}{|c|}{ 42CrMo4 } & \multicolumn{2}{|c|}{ AISI 304} & \multicolumn{2}{|c|}{ AISI 304} \\
\hline Weight (gr) & \multicolumn{2}{|c|}{230.00} & \multicolumn{2}{|c|}{260.00} & \multicolumn{2}{|c|}{1073.27} & \multicolumn{2}{|c|}{1887.99} \\
\hline PROCDSS ITEM COSTS & Calc. & DFMA & Calc. & DFMA & Calc. & DFMA & Calc. & DFMA \\
\hline Core manufacturing & 0.00 & 0.00 & 0.00 & 0.00 & 3.27 & 5.78 & 7.03 & 17.15 \\
\hline Pattern manufacturing & 1.97 & 0.83 & 2.03 & 0.84 & 5.24 & 2.99 & 7.68 & 5.28 \\
\hline Pattern assembly & 0.00 & 0.00 & 0.00 & 0.00 & 0.00 & 0.00 & 0.00 & 0.00 \\
\hline Cluster assembly & 1.80 & 0.55 & 2.02 & 0.61 & 6.92 & 1.88 & 10.42 & 3.65 \\
\hline Dissolving core & 0.00 & 0.00 & 0.00 & 0.00 & 0.01 & 0.00 & 0.02 & 0.00 \\
\hline Cleaning and etching & 0.00 & 0.00 & 0.00 & 0.00 & 0.00 & 0.02 & 0.01 & 0.03 \\
\hline Investing the cluster & 1.26 & 1.61 & 1.41 & 1.77 & 4.95 & 5.61 & 8.41 & 9.11 \\
\hline Melt out & 0.10 & 0.04 & 0.11 & 0.04 & 0.38 & 0.15 & 0.64 & 0.30 \\
\hline Sintering & 0.37 & 0.20 & 0.41 & 0.23 & 1.46 & 0.81 & 2.47 & 1.63 \\
\hline Melting & 3.92 & 2.88 & 4.38 & 3.20 & 35.99 & 26.43 & 52.56 & 44.00 \\
\hline Break out & 0.02 & 0.01 & 0.02 & 0.01 & 0.07 & 0.03 & 0.11 & 0.07 \\
\hline Blast cleaning & 0.02 & 0.01 & 0.02 & 0.01 & 0.06 & 0.02 & 0.11 & 0.04 \\
\hline Cut off & 0.06 & 0.23 & 0.06 & 0.25 & 0.09 & 1.20 & 0.15 & 2.16 \\
\hline Total cost & 9.52 & 6.37 & 10.45 & 6.97 & 58.44 & 44.92 & 89.62 & 83.43 \\
\hline Actual value & \multicolumn{2}{|c|}{10.41} & \multicolumn{2}{|c|}{11.13} & \multicolumn{2}{|c|}{58.37} & \multicolumn{2}{|c|}{100.00} \\
\hline Dev. Total vs Actual & $9 \%$ & $39 \%$ & $6 \%$ & $37 \%$ & $0 \%$ & $23 \%$ & $10 \%$ & $17 \%$ \\
\hline
\end{tabular}

Figure 7. Foundry No. 2 components cost analysis

\section{Conclusions and future work}

Investment casting is a manufacturing process consisting of multiple phases (e.g.: pattern manufacturing, cluster assembling, melting, etc.), that employs several materials (e.g.: wax, stucco, refractory earths, metal, etc.), machines (e.g.: furnaces, autoclaves, injection presses, etc.) and tooling. This process can be realized employing different production lines (manual vs automatic) all over the world. An analytical cost model for such process is characterized by hundreds of different process parameters, elaborated by hundreds of equations and thousands of database values. This amount of required information makes very complex defining a robust cost model for investment casting. This paper wants to improve the cost model proposed in (Boothroyd et al., 2011), the gold standard for cost estimation of investment casting, to improve its robustness for manual production lines, since it seems more fitting with automatic ones.

The manuscript presents the investment casting phases that have been analysed in cooperation with two different foundries, with the aim to improve reliability of the cost estimated using the reference model. The paper illustrates improved equations, data and considerations to be used for cost estimation.

The cost model achieved after the research activities was tested in cooperation with the foundries involved in this project. Two datasets of results were obtained, one for each foundry. Each dataset consists of (i) cost estimated with the proposed model, (ii) cost estimated with the reference model and (iii) actual cost of the foundries. For the first dataset (manual production) the cost estimated by the proposed model deviates from the actual cost of around $14 \%$. This model behaves better than the same one considered as baseline, for which deviation is around 56\%. For the second dataset (automatic production), deviation between estimated (using the proposed mode) and actual cost is around 6\%, better the reference mode, which has a deviation of around $29 \%$. 
Future work will aim to evaluate the robustness of this cost model for tiny components (less than 50 grams), commonly used in footwear, jewels and clothing sectors. Indeed, validation has been carried out considering only components heavier than 200 grams. Furthermore, such analytical model can be used for defining a parametric one, that can be used earlier in the design phase. Sensitivity analyses proposed in this paper will be used for correlating product and process cost drivers with the manufacturing cost, hence establishing parametric equations and curves.

\section{Acknowledgement}

Authors are very grateful to Eng. Marco Bigliardi and Dr. Gianna Bigliardi (Microfound Srl), Eng. Eugenio Manni (Lario Industry Srl) and Eng. Giulio Marcello Lo Presti, Eng. Emanuele Checcacci, Eng. Irene Martinelli, Eng. Francesco Pescatori and Eng. Antonio Pumo (Baker Hughes) and Eng. Giulia Torregiani (Università Politecnica delle Marche) for their technical contribution about the investment casting process.

\section{References}

Arundacahawat, P., Roy, R. and Al-Ashaab, A. (2013), "An analogy based estimation framework for design rework efforts", Journal of Intelligent Manufacturing, Vol. 24 No. 3, pp. 625-639. https://doi.org/ 0.1007/s10845-011-0605-6

Boothroyd, G., Dewhurst, P. and Knight, W.A. (2011), "Product Design For Manufacture and Assembly", 3rd Edition CRC Press.

Boothroyd, G. and Radovanovic, P. (1989), "Estimating the Cost of Machined Components During the Conceptual Design of a Product",CIRP Annals, Vol. 38 No. 1, pp. 157-160. https://doi.org/10.1016/S00078506(07)62674-2

Campi, F. et al. (2019), "Using design geometrical features to develop an analytical cost estimation method for axisymmetric components in open-die forging", Procedia CIRP, Vol. 84, pp. 656-661. https://doi.org/ 10.1016/j.procir.2019.04.324

Chronister, T.G. (1975), "The Cost of making castings", Casting Engineering, Vol. 7 No. 4, pp. 8-11.

Creese, R. and Adithan, M. (1992), "Estimating and Costing for the Metal Manufacturing Industries", 1st Edition CRC Press. https://doi.org/10.1201/9780429246654

Das, S.K., Datla, V. and Samir, G. (2000), "DFQM - An approach for improving the quality of assembled products", Int. J. Product Res, Vol. 38 No. 2, pp. 457-477. https://doi.org/10.1080/002075400189527

Duverlie, P. and Castelain, J.M. (1999), "Cost estimation during design step: parametric method versus case based reasoning method", International Journal of Advanced Manufacturing Technology, Vol. 15 No. 12, pp. 895-906. https://doi.org/10.1007/s001700050147

Fagade, A. and Kazmer D. (2000), "Early cost estimation of injection molded components", Journal of Injection Molding Technology, Vol. 4 No. 3, pp. 97-106.

Farineau, T. et al. (2001), "Use of parametric models in an economic evaluation step during the design phase" International Journal of Advanced Manufacturing Technology, Vol. 17 No. 2, pp. 79-86. https://doi.org/ $10.1007 / \mathrm{s} 001700170195$

Favi, C., Germani, M. and Mandolini, M. (2017), "Analytical Cost Estimation Model in High Pressure Die Casting", Procedia Manufacturing, Vol 11, pp. 526-535. https://doi.org/10.1016/j.promfg.2017.07.146

García-Crespo, Á. et al. (2011), "A review of conventional and knowledge based systems for machining price quotation", Journal of Intelligent Manufacturing, Vol. 22 No. 6, pp. 823-841. https://doi.org/10. 1007/s10845-009-0335-1

Jung, J.Y. (2002), "Manufacturing cost estimation for machined parts based on manufacturing features", Journal of Intelligent Manufacturing, Vol. 13 No. 4, pp. 227-238. https://doi.org/10.1023/A:1016092808320

Kulkarni, A. (1988), "Cost control in foundries through innovation", Indian Foundry Journal, Vol. 34 No. 1, pp. 21-25.

Luong, L. and Spedding, T. (1995), "An integrated system for process planning and cost Estimation in hole making" International Journal of Advanced Manufacturing Technology, Vol. 10 No. 6, pp. 411-415. https://doi.org/10.1007/BF01179405

Maciol A. (2017), "Knowledge-based methods for cost estimation of metal casts", International Journal of Advanced Manufacturing Technology, Vol. 91 No. 1-4, pp. 641-656. https://doi.org/10.1007/s00170-0169704-z

Martinelli, I. et al. (2019), "Cost Estimation Method for Gas Turbine in Conceptual Design Phase”, Procedia CIRP, Vol. 84, pp. 650-655. https://doi.org/10.1016/j.procir.2019.04.311

Niazi, A. et al. (2005), "Product Cost Estimation: Technique Classification and Methodology Review" Journal of Manufacturing Science and Engineering, Vol. 128 No. 2, pp. 563-575. https://doi.org/10.1115/1.2137750 
Ou-Yang, C. and Lin, T.S. (1997), "Developing an Integrated Framework for Feature-Based Early Manufacturing Cost Estimation” International Journal of Advanced Manufacturing Technology, Vol. 13 No. 9, pp. 618-629. https://doi.org/10.1007/BF01350820

Pattnaik, S., Karunakar, B.D. and Jha, P.K. (2012), "Developments in investment casting process-A review" Journal of Materials Processing Technology, Vol. 212 No. 11, pp. 2332-2348. https://doi.org/10.1016/ j.jmatprotec.2012.06.003

Rush, C. and Roy, R. (2001), "Expert judgement in cost estimating: modelling the reasoning process" Concurrent Engineering, Vol. 9 No. 4, pp. 271-284. https://doi.org/10.1177/1063293X0100900404

Sajid, M. et al. (2018), "Manufacturing feature-based cost estimation of cast parts" China foundry, Vol. 15 No. 6, pp. 464-469. https://doi.org/10.1007/s41230-018-8084-4

Shehab, E.M. and Abdalla, H.S. (2002), "An Intelligent Knowledge-Based System for Product Cost Modeling”, International Journal of Advanced Manufacturing Technology, Vol. 191 No. 1, pp. 49-65. https://doi.org/ 10.1007/PL00003967

Sohlenius, G. (1992), “Concurrent Engineering”. CIRP Annals - Manufacturing Technology, Vol. 41 No. 2, pp. 645-655. https://doi.org/10.1016/S0007-8506(07)63251-X

Ulrich, K.T. and Eppinger, S.D. (2003), "Product Design and Development", Third Edition, McGraw-Hill Inc., USA.

Verlinden, B. et al. (2008), "Cost estimation for sheet metal parts using multiple regression and artificial neural networks: A case study" International Journal of Production Economics, Vol. 111 No. 2, pp. 484-492. https://doi.org/10.1016/j.ijpe.2007.02.004

Wang, H., Zhou, X.H. and Ruan, X.Y. (2003), "Research on injection mould intelligent cost estimation system and key technologies", International Journal of Advanced Manufacturing Technology, Vol. 21 No. 3, pp. 215-222. 